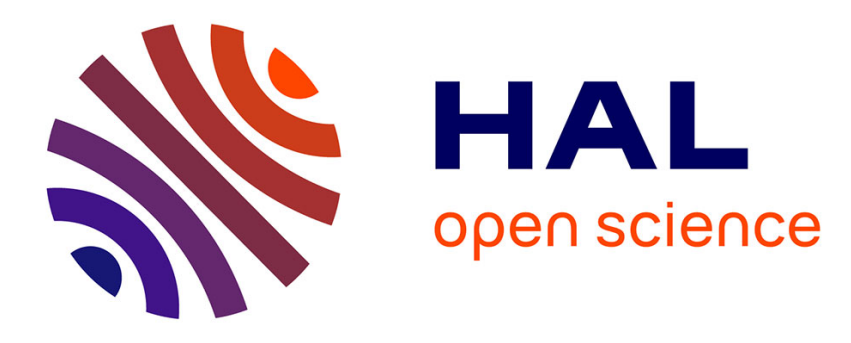

\title{
Study of different structures of the knee joint for a planar bipedal robot
}

\author{
Arnaud Hamon, Yannick Aoustin
}

\section{To cite this version:}

Arnaud Hamon, Yannick Aoustin. Study of different structures of the knee joint for a planar bipedal robot. Humanoids2009, Dec 2009, Paris, France. pp.113-120. hal-00422259

\section{HAL Id: hal-00422259 \\ https://hal.science/hal-00422259}

Submitted on 14 Jan 2010

HAL is a multi-disciplinary open access archive for the deposit and dissemination of scientific research documents, whether they are published or not. The documents may come from teaching and research institutions in France or abroad, or from public or private research centers.
L'archive ouverte pluridisciplinaire $\mathbf{H A L}$, est destinée au dépôt et à la diffusion de documents scientifiques de niveau recherche, publiés ou non, émanant des établissements d'enseignement et de recherche français ou étrangers, des laboratoires publics ou privés. 


\title{
Study of different structures of the knee joint for a planar bipedal robot
}

\author{
A. Hamon and Y. Aoustin
}

\begin{abstract}
Usually, the bipedal robots use knee joints with only one degree of freedom. However, several biomechanical researches have proved the human knee joint is a very complex articulation with more than one degree of freedom. Indeed, in the sagittal plain the human knee articulation allows a rolling and a sliding of the thigh on the shin. Moreover, we can use for a bipedal robot a closed structure, which gives an additional degree of freedom in translation for the knee joint in the sagittal plane. Therefore, the aim of this paper is to study two different bipedal robots. One with single axis knee joints and an other with four-bar knee joints. These bipeds are defined in the sagittal plain and are composed of two shins, two thighs and a trunk but they have no feet or arms. In the two cases, we use a parametric optimization method to produce reference cyclic trajectories in order to compare the energy consumption of the bipedal robots. A Poincaré return map is defined for the walking gaits of the bipedal robots in order to study the influence of the two different knees on their orbital stability.
\end{abstract}

\section{INTRODUCTION}

Lot of papers are devoted to the definition of reference walking trajectories for bipedal robots in two or three dimensions, [1], [2], [3], [4], [5]. These works proved the possibility to define by optimization more efficient trajectories from the point of view of energy consumption for a bipedal robot. However, the necessary energetic autonomy of the bipedal robots could be obtained by a better comprehension of the human's lower limbs. Indeed, most biomechanical researches have been interested on the study of the human knee joint [6], [7], [8], [9]. There studies defined precisely the different movements of the knee. The main characteristic of this articulation is a very complex joint, which is composed of non-matching surfaces, with six degrees of freedom [10]. This articulation allows rolling and sliding movements of the femur on the shin in the sagittal plane. In opposition, most bipedal robots, like HRP-2 [11], Rabbit [12], Wabian2 [13], have knee joints with only one degree of freedom. We can note G. Gini et al. in [14] have developed an anthropomorphic leg for the robot LARP with a new type of knee, which allows a rolling and a sliding of the femur on the shin. Moreover F.Wang et al., [15], have developed a bipedal robot with two different legs. The first with a single axis knee joint and the second with a parallel knee structure. This simple structure called four-bar knee, reproduce a part of the human knee movement. Indeed, this structure allows a coordinated movement of rotation and translation of the thigh on the shin, but prevent the dislocation of the knee joint as the cruciate ligaments for the human knee [6], [16], [17].

A. Hamon is with IRCCyN, UMR 6597, Ecole Centrale de Nantes, Université de Nantes, France (e-mail: Arnaud.Hamon@irccyn.ec-nantes.fr).

Y. Aoustin is with IRCCyN, UMR 6597, Ecole Centrale de Nantes, Université de Nantes, France (e-mail: Yannick.Aoustin@irccyn.ec-nantes.fr).
So the interests of this structure are a polycentric center of rotation like in the human knee joint, a higher foot clearance for a smaller flexion angle than a single axis knee and a better reaction force of the ground to keep the stability during a walking gate. So we purpose to prove the interest of a complex knee joint in term of energy consumption for a planar bipedal robot. We choose to study two different planar bipedal robots. One with revolute knee joints and the other with four-bar knees. The mass and inertial properties of the different segments are chosen to correspond to the human characteristics [7]. The two robots are composed of a trunk, two shins and two thighs but have no feet or arms. We use these very simple robots to center the study on the energetic consumption of the knee joint. In each case, we pose a parametric optimization problem to obtain walking cyclic gait. The steps are composed of single support phases separated by an impulsive impact. The reference trajectories for the actuated joints are defined like polynomial functions of an unactuated angle, which is the orientation of the support leg versus the inertial frame, to solve the problem of under actuation of the bipedal robot in single support phase. The four-bar knee is not a new structure. But the main contribution of this paper is the study of the energetic interest of the 4-bar for the knee joint versus to a revolute joint by using of an optimization method. Moreover a criterion of stability with the Poincaré return map [12] is computed to study the influence of the four-bar knee on the orbital stability.

So, we will be presented in section II the different bipedal robots. Section III presents the structure and physical data of the two bipedal robots. In section IV, the problem of the trajectory optimization is addressed. Numerical tests are analyzed in section V. Finally, section VI offers our conclusions and perspectives.

\section{Presentation of the DifFEREnT Robots}

The first bipedal robot, figure (1), is a planar bipedal robot with point feet. All joints are revolute and have only one degree of freedom. The second bipedal robot, figure (2), uses four-bar knees. These knees are composed of a closed structure that allows a synchronized movement of the knee in translation and rotation. The dimensions of both biped are chosen to respect the human's characteristics. The lengths, masses and inertia moments for the segments of the bipeds are computed from a table of anthropomorphic data (see [7]), obtained by W. Dempster in [18]. For the biped with four-bar knee joints the whole mass is $67 \mathrm{~kg}$, its height is $1.62 \mathrm{~m}$. The masses and the lengths of its five links are: $m_{1}=m_{4}=3.48 \mathrm{~kg}, m_{2}=m_{3}=7.5 \mathrm{~kg}, m_{5}=43.35 \mathrm{~kg}$. 
The length of the shins are $l_{1}=l_{4}=0.497 \mathrm{~m}$ (between the ankle and the base of the 4 bar-knee structure), the length of the thighs are $l_{2}=l_{3}=0.497 \mathrm{~m}$, and the length of the trunk is $l_{5}=0.625 \mathrm{~m}$. The distances between the knee joint and the center of mass of the shin are: $s_{1}=s_{4}=0.215 \mathrm{~m}$; between the hip joint and the center of mass of thigh: $s_{2}=$ $s_{3}=0.215 \mathrm{~m}$; between the hip joint and the center of mass of the trunk: $s_{5}=0.386 \mathrm{~m}$. The inertia moments around the mass center of each links are: $I_{1}=I_{4}=0.058 \mathrm{~kg} \cdot \mathrm{m}^{2}$, $I_{2}=I_{3}=0.145 \mathrm{~kg} \cdot \mathrm{m}^{2}, I_{5}=2.137 \mathrm{~kg} \cdot \mathrm{m}^{2}$. The length of the different parts of the four-bar knee are $l_{a}=0.0425 \mathrm{~m}$, $l_{b}=0.03 \mathrm{~m}, l_{c}=0.025 \mathrm{~m}, l_{d}=0.051 \mathrm{~m}, l_{e}=0.004 \mathrm{~m}$ (see figure (3)) and the masses are $m_{b}=0.25 \mathrm{~kg}, m_{d}=0.35 \mathrm{~kg}$.

For the second bipedal robot with revolute knee joints, we use the same characteristics of weight and length of the different bodies but to compare the results between the two robots we need to change a few numbers of values to take into account the dimension of the four-bar structure. So, the length of the shins are $l_{1}=l_{4}=0.501 \mathrm{~m}$ and the mass of the thighs are $m_{2}=m_{3}=8.1 \mathrm{~kg}$.

\section{BIPED MODEL}

This part is devoted to the definition of a dynamic model for each robot in single support phase. First, we present the dynamic model of the robot with a single axis knee and the model of the impulsive impact. Finally, we present the differences with the bipedal robot using a four-bar knee structure.

\section{A. Model of the biped with single axis knee joints}

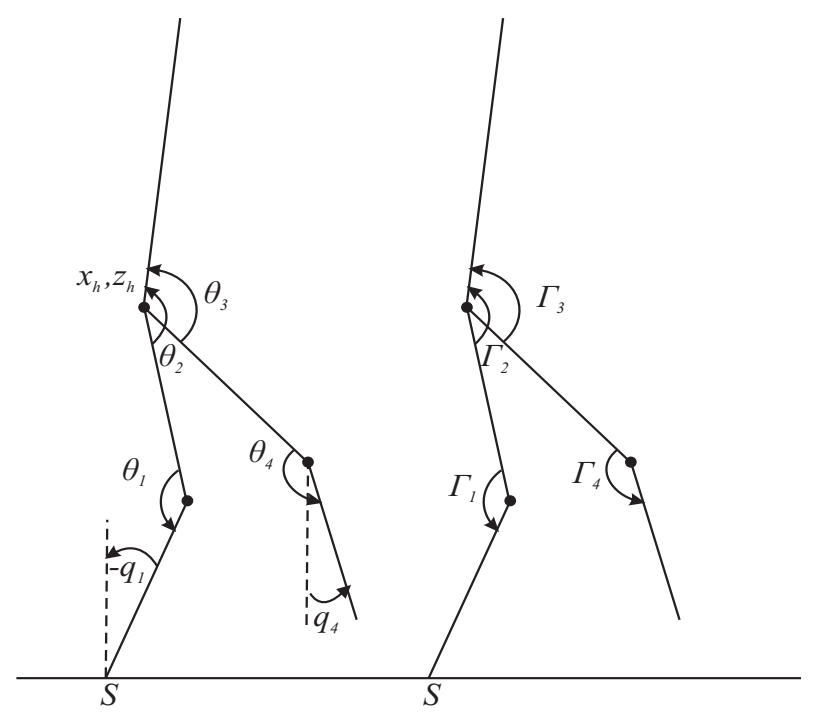

Fig. 1. Diagram of the planar bipedal robot with single axis knee joints: generalized coordinates, applied torques.

1) Dynamic model: To define the dynamic model of the planar biped, which is depicted in figure (1), let us introduce vector $\Theta_{a}=\left(\theta_{1}, \theta_{2}, \theta_{3}, \theta_{4}\right)^{* 1}$ of the actuated angles and the unactuated angle $q_{1}$ corresponding to the angle between the

\footnotetext{
${ }^{1}$ Notation ${ }^{*}$ means transposition
}

vertical axis and the shin. We use the vector $\Theta=\left(\Theta_{a}^{*}, q_{1}\right)^{*}$ to define the dynamic model of the bipedal robot. The two Cartesian coordinates $x_{h}, z_{h}$ are the abscissa and the ordinate of the hip joint. The vector $\Gamma=\left(\Gamma_{1}, \Gamma_{2}, \Gamma_{3}, \Gamma_{4}\right)^{*}$ regroups the applied torques in the joints and $R_{j}=\left(R_{j x}, R_{j z}\right)$, the ground reaction applied to the point foot $j$. If we consider that the fixed leg tip is linked on the ground with a pivot joint, we can calculate the dynamic model of the bipedal robot such as,

$$
A\left(\Theta_{a}\right) \ddot{\Theta}+C(\Theta, \dot{\Theta}) \dot{\Theta}+G(\Theta)=D_{\Gamma} \Gamma
$$

As the kinetic energy of the planar biped is invariant under a rotation of the world frame [19], the $5 \times 5$ positive inertia matrix $A=A\left(\Theta_{a}\right)$ is independent of variable $q_{1}$ which defines the orientation of the biped. $C(\Theta, \dot{\Theta})$ is the $5 \times 5$ matrix of the centrifugal and Coriolis forces and $G(\Theta)$ is the $5 \times 1$ vector of the gravity forces. $D_{\Gamma}$ is a $5 \times 4$ fixed matrix constituted of zeros and units.

This formulation of the dynamic model is valid only if the stance leg tip does not take off and there is no sliding during the swing phase. In consequence, the contact point of the stance leg tip with the ground is considered as a pivot joint. Then, the vertical component of the ground reaction in the stance leg tip has to be positive and the ground reaction must be inside the friction cone. The ground reaction in the stance leg tip can be calculated by applying the fundamental dynamic principle in the center of mass of the biped:

$$
\left(\begin{array}{l}
R_{1 x} \\
R_{1 z}
\end{array}\right)+\left(\begin{array}{l}
R_{2 x} \\
R_{2 z}
\end{array}\right)=m\left(\begin{array}{c}
\ddot{x}_{g} \\
\ddot{z}_{g}
\end{array}\right)+m\left(\begin{array}{l}
0 \\
g
\end{array}\right)
$$

Here, $\mathrm{m}$ is the biped's mass, $\ddot{x}_{g}$ and $\ddot{z}_{g}$ are the components of the acceleration for its center of mass. During the single support phase, reaction $R_{2}$ of the ground on the balance leg tip is null. Then, this equation allows directly to get the reaction force $R_{1}$ of the ground in single support.

2) Model of the impulsive impact: The impact model is deduced from the dynamic model in double support, when we assume that the acceleration of the bipedal robot and the reaction forces are Dirac delta-functions. The dynamical model in double support can be written:

$$
A_{e}\left(\Theta_{a}\right) \ddot{X}+C_{e}(X, \dot{X}) \dot{X}+G_{e}(X)=D_{\Gamma e}+J_{R_{1}}^{*} R_{1}+J_{R_{2}}^{*} R_{2}
$$

Here, $X=\left[\theta_{1}, \theta_{2}, \theta_{3}, \theta_{4}, q_{1}, x_{h}, z_{h}\right]^{*}$ where $x_{h}, z_{h}$ are the coordinates of the hip joint. Matrices $J_{R 1}^{*}$ and $J_{R 2}^{*}$ are the transposed jacobian matrices in leg tips 1 and 2. These matrices and $D_{\Gamma}$ allow to take into account the ground reaction and torques in the dynamic model. The impact is assumed inelastic without rebound. That is why the velocity of the swing foot impacting the ground is zero after impact.

Two cases are possible after an impact: the rear foot takes off the ground or both feet remain on the ground.

In the first case, the vertical component of the velocity of the taking-off leg tip just after an impact must be directed upwards and the impulsive ground reaction in this leg tip equals zeros $\left(R_{1}=0\right)$. In the second case, the velocity of the rear leg tip has to be zero just after impact. The ground produces impulsive forces in both leg tips. Then, the vertical 
component of the impulsive ground reaction in the rear leg tip (as in the foreleg tip) has to be directed upwards. In [2] $\mathrm{S}$. Miossec has studied the problem of the impact for the same type of planar bipedal robot without feet and proved a swing leg with zero velocity just before impact is the only valid solution to obtain a finite time double support phase. There is no impact, the reaction forces on the two leg tips are zero and the velocity of the two leg tips after impact is null. Then, only walking cyclic gaits with single support phases and impacts are considered. Thus, the impact model is (see [20])

$$
\begin{array}{r}
A_{e}\left(\Theta_{a}\right)\left(\dot{X}^{+}-\dot{X}^{-}\right)=J_{R} I_{R} \\
J_{R}{ }^{*} \dot{X}^{+}=0
\end{array}
$$

These relations form a matrix system which helps to determine the impulse forces $I_{R}$ and the velocity vector of the biped after impact $\dot{X}^{+}$in function of the velocity vector of the biped just before the impact $\dot{X}^{-}$.

\section{B. Modelling of the robot with four-bar knee joints}

This part is devoted to the definition of a dynamic model for the robot with four-bar knees. The most important part in this section is the study of the four-bar structure (see figure (3)). So, we will present the dynamic model of this robot and the kinematic constraints which limit the number of degrees of freedom of the robot.

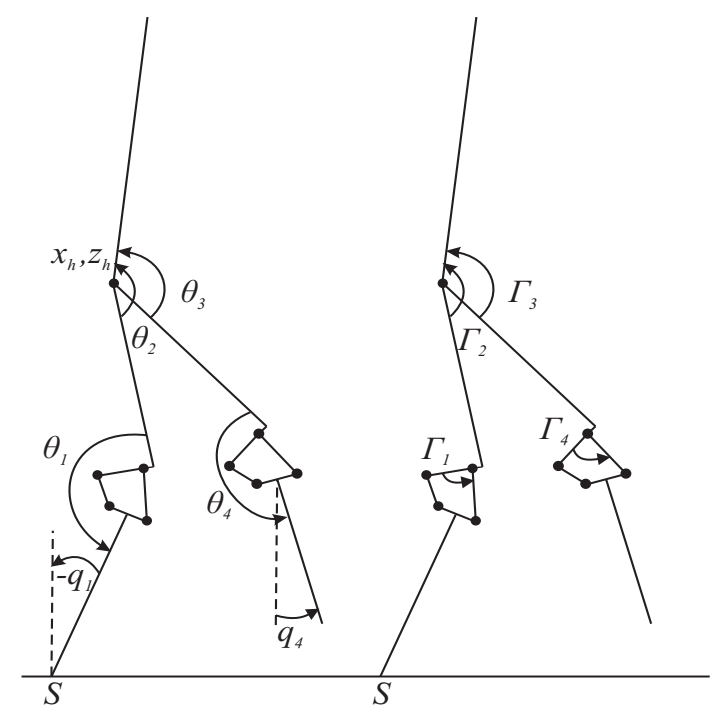

Fig. 2. Diagram of the bipedal robot with a four-bar knee joint in sagittal plane : relative coordinates, applied torques.

1) Dynamic model of the robot with four-bar knees: We have introduced for the robot with single axis knees, the relative angles between the different parts of the robot, but for the robot with four-bar knees, it is necessary to add four angles to represent the robot. So, to define the dynamic model of this robot, we introduce vector $\Theta_{a}=\left(\theta_{1}, \theta_{2}, \theta_{3}, \theta_{4}\right)$ of the actuated angle and $q_{g}=\left(q_{g_{11}}, q_{g_{12}}, q_{g_{21}}, q_{g_{22}}\right)^{*}$ which is the vector of the absolute angles of the four-bar for both knee. $\Theta=\left[\Theta_{a}, q_{1}, q_{g}\right]^{*}$ is the vector of the articular variables of the planar biped.

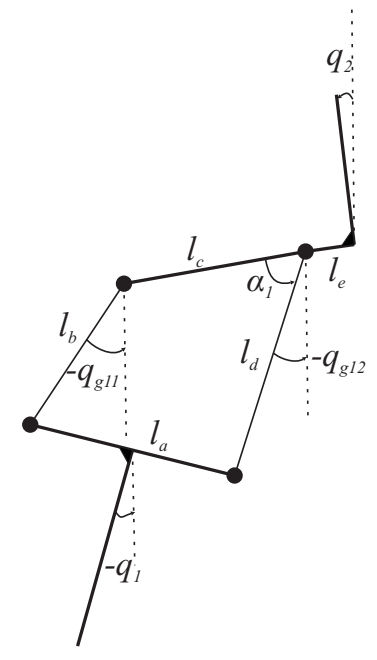

Fig. 3. Detail of the four-bar knee joint on the stance leg

So, with this notation the dynamic model of this biped is:

$$
A\left(\Theta_{a}\right) \ddot{\Theta}+C(\Theta, \dot{\Theta}) \dot{\Theta}+G(\Theta)=D_{\Gamma} \Gamma
$$

2) Computation of the $D_{\Gamma}$ matrix: For the robot with four-bar knees, the angles which are actuated on the knees are not $\theta_{1}$ and $\theta_{4}$ like in the single axis case but the angles note $\alpha_{1}$ and $\alpha_{2}$ for the legs 1 and 2 respectively (see figure (3) for leg 1). With our choice of notation for the dynamic model, matrix $D_{\Gamma}$ is different from in section III-A. It is necessary to use the virtual work principle [2] to obtain this matrix. So we present in this part, the principle of the computation. Let us introduce the virtual work of the actuators for this robot with our notation:

$$
\delta W=\delta \alpha_{1} \delta \Gamma_{1}+\delta \theta_{2} \delta \Gamma_{2}+\delta \theta_{3} \delta \Gamma_{3}+\delta \alpha_{2} \delta \Gamma_{4}
$$

with:

$$
\begin{aligned}
& \delta \alpha_{1}=\delta \theta_{1}-\delta q_{1}+\delta q_{g_{12}} \\
& \delta \alpha_{2}=\delta \theta_{4}-\delta q_{4}+\delta q_{g_{22}}
\end{aligned}
$$

Here, $\delta \alpha_{i}$ and $\delta \theta_{i}$ are the virtual articular displacements of the angles $\alpha_{i}$ and $\theta_{i} . q_{4}$, which is the absolute angle of the swing leg's shin, is not a variable of $\Theta$, but we can obtain the following relation between $q_{4}$ and $\Theta$ :

$$
\delta \alpha_{2}=\delta \theta_{1}-\delta q_{1}-\delta \theta_{2}+\delta \theta_{3}+\delta q_{g_{22}}
$$

and we have:

$$
D_{\Gamma}=\frac{\partial}{\partial \delta \Gamma}\left(\frac{\partial \delta W}{\partial \delta \Theta}\right)
$$


Finally :

$$
D_{\Gamma}=\left(\begin{array}{cccc}
1 & 0 & 0 & 1 \\
0 & 1 & 0 & -1 \\
0 & 0 & 1 & 1 \\
0 & 0 & 0 & 0 \\
-1 & 0 & 0 & -1 \\
0 & 0 & 0 & 0 \\
1 & 0 & 0 & 0 \\
0 & 0 & 0 & 0 \\
0 & 0 & 0 & 1
\end{array}\right)
$$

3) Kinematic constraints on the four-bar knees: An important difference between the first robot is on the kinematic constraints. Indeed, we can see with the previous notation the robot has nine degrees of freedom, but four actuators. If we want to reduce the degree of under actuation at one, it is necessary to choose four equations of constraints. For each knee, we have introduced two new variables but the constraints of closing of the loop give two equations for each knee.

$$
\begin{aligned}
& -l_{a} \cos \left(q_{1}\right)-l_{b} \sin \left(q_{g_{11}}\right)+l_{c} \cos \left(q_{2}\right)+l_{d} \sin \left(q_{g_{12}}\right)=0 \\
& -l_{a} \sin \left(q_{1}\right)+l_{b} \cos \left(q_{g_{11}}\right)+l_{c} \sin \left(q_{2}\right)-l_{d} \cos \left(q_{g_{12}}\right)=0 \\
& -l_{a} \cos \left(q_{4}\right)-l_{b} \sin \left(q_{g_{21}}\right)+l_{c} \cos \left(q_{3}\right)+l_{d} \sin \left(q_{g_{22}}\right)=0 \\
& -l_{a} \sin \left(q_{4}\right)+l_{b} \cos \left(q_{g_{21}}\right)+l_{c} \sin \left(q_{3}\right)-l_{d} \cos \left(q_{g_{22}}\right)=0
\end{aligned}
$$

With these four equations, it is possible to obtain the variables $q_{g_{11}}, q_{g_{12}}, q_{g_{21}}, q_{g_{22}}$ in function of $q_{1}, q_{2}, q_{3}, q_{4}$. Moreover, if we derived these four equations we can compute velocities and accelerations of $q_{g_{11}}, q_{g_{12}}, q_{g_{21}}, q_{g_{22}}$ in function of the others. So Finally, this robot has just one degree of under actuation.

\section{OPTIMIZATION}

In this section, we present a methodology to design a parametrized family of cyclic reference trajectories for the two planar bipedal robots. In each case, the evolution of the articulations is defined like polynomial functions of an unactuated angle, which changes monotonically during the step. Moreover, in order to determine a particular solution of motion which minimizes the mechanical energy consumption, a parametric optimization process under constraints is presented.

\section{A. Definition of actuated joints trajectories}

The biped with its stance point feet is underactuated in single support phase. The degree of underactuation equals one. To take into account this problem and to ensure the biped reaches its final desired configuration at the impact, the reference trajectories of the actuated joints $\theta_{j}, j=1$ to 6 are polynomial functions of the unactuated generalized variable $q_{1}$, figures (1) and (2). Indeed, different researches [21], [22], [23] conclude this angle is always monotonically during a single support phase for most of walking gaits. So, this angle could be used instead of time for the bipedal robot. The order of these polynomial functions equals three to prescribed the initial and final values for the joint variables and their velocities.

$$
\theta_{j}\left(q_{1}\right)=a_{0}+a_{1} q_{1}+a_{2} q_{1}^{2}+a_{3} q_{1}^{3} \text { for } \mathrm{j}=1 \text { to } 6 .
$$

Here, the coefficients $a_{i}$, for $i=0$ to 3 , are calculated with the parameters $\theta_{j_{i}}, q_{1_{i}}, \dot{\theta}_{j_{i}}, \dot{q}_{1_{i}}, \theta_{j_{f}}, q_{1_{f}}, \dot{\theta}_{j_{f}}$ and $\dot{q}_{1_{f}}$. Moreover, we can define the velocities such as:

$$
\dot{\theta}_{j}=\frac{\partial \theta_{j}\left(q_{1}\right)}{\partial q_{1}} \dot{q}_{1} \text { for } \mathrm{j}=1 \text { to } 6
$$

and the acceleration :

$$
\ddot{\theta}_{j}=\frac{\partial \theta_{j}\left(q_{1}\right)}{\partial q_{1}} \ddot{q}_{1}+\frac{\partial^{2} \theta_{j}\left(q_{1}\right)}{\partial q_{1}^{2}} \dot{q}_{1}^{2} \text { for } \mathrm{j}=1 \text { to } 6 .
$$

This method is only valid if the behavior of the angle $q_{1}$ is monotonous. So, its derivative $\dot{q}_{1}$ has to be strictly negative here. Indeed, if we denote $\sigma$ as the angular momentum around the fixed point $S$ of the stance foot, we can define the equation:

$$
\sigma=\dot{q}_{1} f\left(q_{1}\right)
$$

where $f\left(q_{1}\right)$ is determined by the fifth line of equation (1) which corresponds to the angle $q_{1}$ such as:

$$
\begin{array}{r}
f\left(q_{1}\right)=A_{q_{1}}\left(\Theta_{a}\right) \frac{\partial \Theta\left(q_{1}\right)}{\partial q_{1}} \\
\frac{\partial f\left(q_{1}\right)}{\partial q_{1}}=A_{q_{1}}\left(\Theta_{a}\right) \frac{\partial^{2} \Theta\left(q_{1}\right)}{\partial q_{1}^{2}}+C_{q_{1}}(\Theta, \dot{\Theta}) \frac{\partial \Theta\left(q_{1}\right)}{\partial q_{1}}
\end{array}
$$

where $A_{q_{1}}$ and $C_{q_{1}}$ are the fifth lines of the inertia matrix and the Centrifugal vector corresponding to the generalized coordinate $q_{1}$. Moreover, the gravity is only the external force which creates a moment, so the angular momentum changes according to the equation:

$$
\dot{\sigma}=-m g\left(x_{g}-x_{S}\right)
$$

where $x_{g}$ and $x_{S}$ are the horizontal positions of the center of gravity of the biped and the contact point respectively. So, we can define the relationship:

$$
\dot{\sigma}=\frac{\partial \sigma}{\partial q_{1}} \dot{q}_{1}=\frac{\partial \sigma}{\partial q_{1}} \frac{\sigma}{f\left(q_{1}\right)}=\frac{1}{2} \frac{\partial \sigma^{2}}{\partial q_{1}} \frac{1}{f\left(q_{1}\right)}
$$

which gives:

$$
\frac{\partial \sigma^{2}}{\partial q_{1}}=-2 m g\left(x_{g}-x_{S}\right) f\left(q_{1}\right)
$$

The integration of this relation gives:

$$
\sigma^{2}-\sigma^{2}(0)=-2 m g \int_{q_{1_{i}}}^{q_{1}}\left(x_{g}-x_{S}\right) f\left(q_{1}\right) d q_{1}
$$

So, we can introduce the variable $\Phi\left(q_{1}\right)$ such as:

$$
\Phi\left(q_{1}\right)=\sigma^{2}-\sigma^{2}(0)=\dot{q}_{1}^{2} f^{2}\left(q_{1}\right)-\dot{q}_{1}^{2} f^{2}\left(q_{1_{i}}\right)
$$

Finally, we obtain:

$$
\dot{q}_{1}^{2}=\frac{\Phi\left(q_{1}\right)+\dot{q}_{i}^{2} f^{2}\left(q_{1_{i}}\right)}{f^{2}\left(q_{1}\right)}
$$


which gives the dynamic of $q_{1}$ :

$$
\dot{q}_{1}= \pm \frac{\sqrt{\Phi\left(q_{1}\right)+\dot{q}_{1 i}^{2} f^{2}\left(q_{1_{i}}\right)}}{f\left(q_{1}\right)}
$$

but $q_{1}$ is a monotone decreasing function, so $\dot{q}_{1}<0$. Moreover, such as $\sigma<0, f\left(q_{1}\right)$ has to be positive to satisfy the constraints of movement and we can conclude [24]:

$$
\dot{q}_{1}=-\frac{\sqrt{\Phi\left(q_{1}\right)+\dot{q}_{1}^{2} f^{2}\left(q_{1_{i}}\right)}}{f\left(q_{1}\right)}
$$

Similarly the dynamic $\ddot{q}_{1}$ is:

$$
\ddot{q}_{1}=\frac{d}{d t}\left(\frac{\sigma}{f\left(q_{1}\right)}\right)
$$

So, we have:

$$
\ddot{q}_{1}=\frac{\dot{\sigma} f\left(q_{1}\right)-\sigma \dot{f}\left(q_{1}\right)}{f^{2}\left(q_{1}\right)}
$$

which leads:

$$
\ddot{q}_{1}=-\frac{m g\left(x_{g}-x_{S}\right)+\dot{q}_{1}^{2} \frac{\partial f\left(q_{1}\right)}{\partial q_{1}}}{f\left(q_{1}\right)}
$$

Introducing, the square velocity $\left(\dot{q}_{1}{ }^{+}\right)^{2}$, it is possible to define a linear Poincaré return map $Q_{n+1}=P\left(Q_{n}\right)$ [25]. The fixed point $Q^{*}=P\left(Q^{*}\right)$ is asymptotically stable if and only if:

$$
\frac{d P}{d Q_{n}} \leq 1
$$

which leads to [12]:

$$
\left|\frac{\sigma^{+}\left(q_{1_{i}}\right)}{\sigma^{-}\left(q_{1_{f}}\right)}\right|=\frac{\left|\sigma^{+}\left(q_{1_{i}}\right)\right|}{\left|\sigma^{-}\left(q_{1_{f}}\right)\right|}=\delta \leq 1
$$

where $\sigma^{-}\left(q_{1_{i}}\right)$ and $\sigma^{+}\left(q_{1_{f}}\right)$ are the angular momentum of the biped in the stance leg tip just before and just after impact. So, we search to decrease the criterion $\delta$ to improve the stability of the gait.

In conclusion, we can calculate a reference trajectory, from the parameters $\Theta_{a_{i}}, q_{1_{i}}, \Theta_{a_{f}}, q_{1_{f}}, \dot{\Theta}_{a_{i}}, \dot{q}_{1_{i}}, \dot{\Theta}_{a_{f}}, \dot{q}_{1_{f}}$, in function of $q_{1}, \dot{q}_{1}$ and $\ddot{q}_{1}$ and, with the direct dynamic model (1), the needed torques to realize this trajectory. To obtain a walking cyclic gait composed of single supports and impacts we can reduce the number of parameters. For that we take into account the exchange of legs between two steps with the relations into the different parameters. Indeed, the resolution of the impact and the inversion of the legs between two steps allow to reduce the number of parameters. Moreover, we can solve the inverse geometrical model of the leg in the limit configuration of the end of step to reduce of one the set of parameters.

\section{B. The problem of trajectories optimization}

In this section, we will present a parametric optimization process to obtain the best solution of parameters, which minimizes the energy consumption of the trajectory. We use the SQP method (Sequential Quadratic Programming) [26], [27] with the fmincon function of Matlab (C) which allows to solve a problem of optimization under linear and non linear constraints. So, we have to define a criterion, the parameters of the optimization and different technological and physical constraints to obtain a walking gait.

1) The criterion: Many criteria can be used to produce an optimal trajectory. In [28] the authors used a criterion to minimize the effort of control. K. Mombaur in [29] proposed to consider the stability like a criterion of optimization. To obtain a trajectory, which minimizes the energy cost, we use a criterion proportional to the mechanical energy to cover the distance $d$ :

$$
C_{W}=\frac{1}{d} \int_{q_{1_{i}}}^{q_{1_{f}}} \sum_{n=1}^{4}\left|\Gamma_{i} \dot{\theta}_{n}\right| d q_{1}
$$

Indeed if we suppose the actuator can not restore the energy, the mechanical energy for the gait is the integral of the absolute value of the joint mechanical power.

2) The constraints of the optimization problem: To obtain a physical gait we need to introduce lot of constraints. We define two types of constraints:

- The necessary constraints which ensure a valid walking gait. The first constraint is linked to the existence of $\dot{q}_{1}$ and $\ddot{q}_{1}$. So we set the constraint:

$$
\phi\left(q_{1}\right)+f\left(q_{1_{i}}\right)^{2} \dot{q}_{1_{i}}^{2}>0
$$

with :

$$
f\left(q_{1}\right)>0
$$

The secondary constraint ensures the support leg tip does not take off or slide on the ground. So, the ground reaction force is inside a friction cone, defined by the friction coefficient $f$ :

$$
\left\{\begin{array}{l}
\max \left(-f \cdot R_{z}-R_{x}\right) \leq 0 \\
\max \left(-f \cdot R_{z}+R_{x}\right) \leq 0
\end{array}\right.
$$

Moreover, we can introduce a constraint on the ground reaction at the impact:

$$
f I_{R_{z}}>\left|I_{R_{x}}\right|
$$

The third constraint allows ensuring the non penetration of the swinging point feet in the ground. For that we define a polynomial function for the minimum altitude of the swing leg tip in function of its horizontal position:

$$
z(x)=a_{0}+a_{1} x+a_{2} x^{2}+a_{3} x^{3}+a_{4} x^{4}
$$

Here the coefficients of the polynomial function are chosen to satisfy the equation:

$$
z(0)=0 \quad z(d)=0 \quad z\left(\frac{d}{2}\right)=h \quad \dot{z}(0)=0 \quad \dot{z}(d)=0
$$

where $h$ is the maximum value of the polynomial function at the middle of the step. So the altitude of the transfer leg tip has to be greater or equal to the function $z(x)$.

- The unnecessary constraints which ensure a technological realistic gait. First we introduce mechanical stops on 
the angles $\Theta$. Moreover, we can limit the torques with a constraint, which sets a template of the maximum torque of the motor in function of the velocity.

\section{RESULTS}

In this part we present the result of simulation obtained for the two planar bipedal robots. In each case, we compare the energy consumption in function of the walking rate. Moreover we compare the criterion of stability $\delta$ introduced in the previous part. Finally we explain the difference on the energy consumption by comparison of the power uses on the knee support leg.

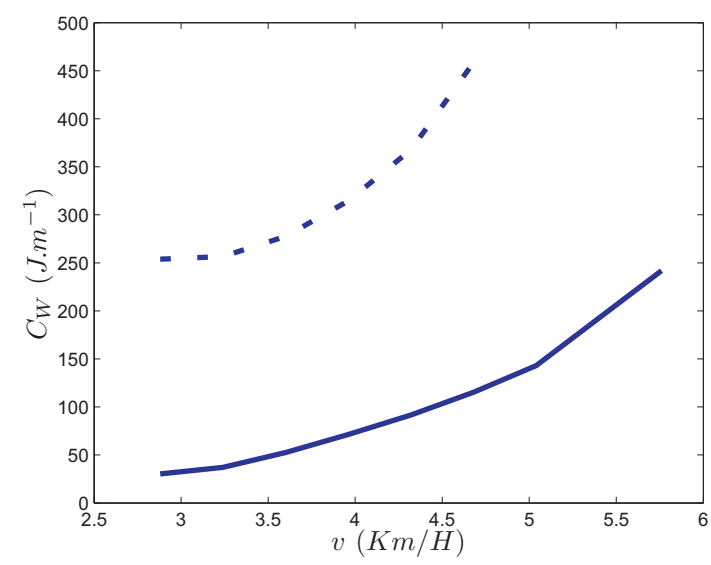

Fig. 4. Evolution of the criterion in function of the walking rate for both bipedal robots: biped with single axis knees (dashed line), robot with fourbar knees (straight line).

Figure (4) gives the evolution of the optimal criterion for the different structures of the knee. The energy cunsomption is less with a biped equiped with four-bar knees than with single joint revolute knees. In the same time, figure (5) gives the evolution of the criterion of stability $\delta$ in function of the walking rate and shows the four-bar knee structure decreases the stability.

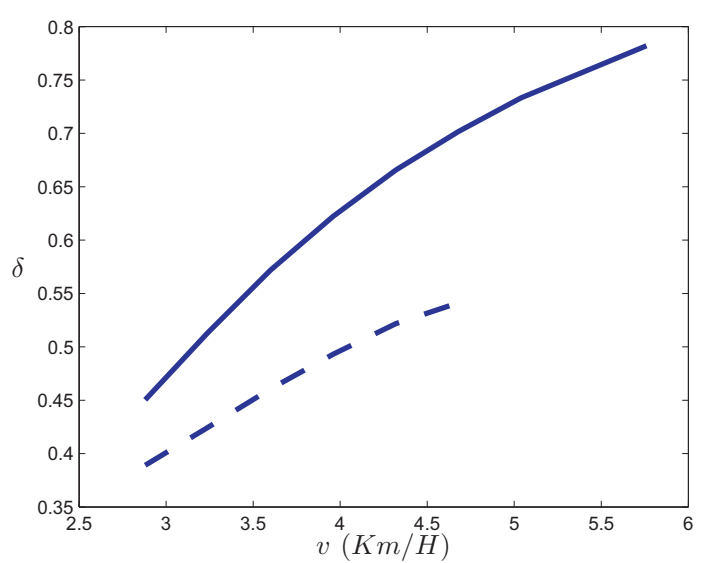

Fig. 5. Evolution of the stability in function of the walking rate for both bipedal robots: biped with single axis knees (dashed line), robot with fourbar knees (straight line).

In order to explain the difference of energy consumption between the two bipedal robots, we study the mechanical energy produced on the knee of the supporting leg in both cases. Let us introduce the mechanical power produced on the support leg with the four-bar knee:

$$
P_{W_{1}}=\left|\Gamma_{1} \dot{\alpha}_{1}\right|
$$

and the mechanical power produced on the single axis knee's support leg:

$$
P_{W_{1}}=\left|\Gamma_{1} \dot{\theta}_{1}\right|
$$

So, we can compare the mechanical energy, which is the integral of the mechanical power for the two robots.

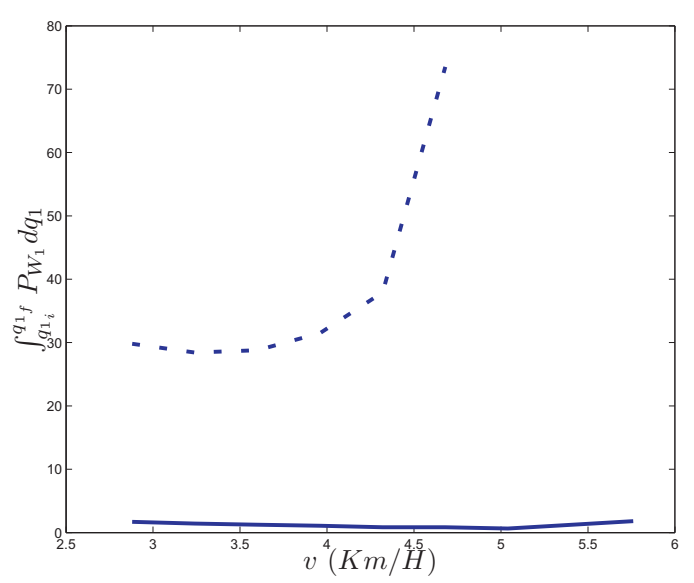

Fig. 6. Energy uses by the knee support leg actuator in function of the walking rate for the robot with four-bar knees (straight line), and for the robot with single axis knees (dashed line).

Figure (6) gives the evolution of the mechanical energy, on knee support leg for both biped in function of the walking rate. We can see the mechanical power developed on the knee support leg is always lower for a four-bar structure. 
Moreover, to understand the effect of the four-bar knee on the energy consumption, we introduce criterion $\psi$, which depicts the ratio between the mechanical power used by an actuator and the total mechanical power:

$$
\psi_{i}=\frac{\int_{q_{1_{i}}}^{q_{1_{f}}} P_{W_{i}} d q_{1}}{C_{W}}
$$

Figures (7) and (8) give the evolution of the criterion $\psi$ for the knee support leg actuator and the hip support leg actuator in function of the walking rate for the two bipeds.

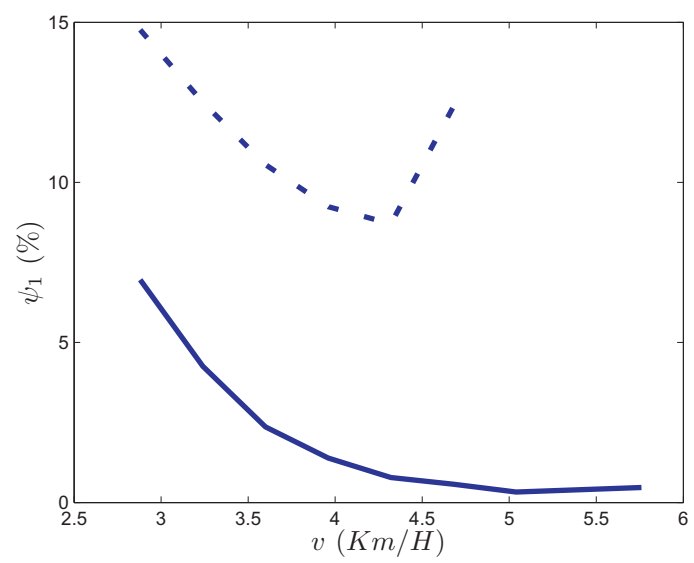

Fig. 7. Evolution of criterion $\psi$ of the knee support leg joint for the robot with four-bar knees (straight line) and for the robot with single axis knee joints (dashed line) in function of the walking rate.

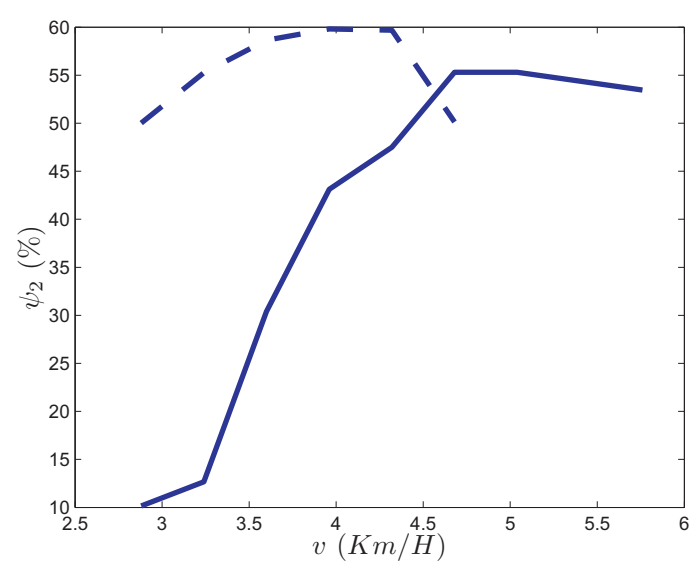

Fig. 8. Evolution of criterion $\psi$ of the hip support leg joint for the robot with four-bar knees (straight line) and for the robot with single axis knee joints (dashed line) in function of the walking rate.

So, for a bipedal robot with single revolute knee joints the energy consumption of the support leg is always greater than $60 \%$ of the total energy consumption. For a biped with four-bar knees, the energy consumption is always lower than $60 \%$ of the total energy consumption. There is an important reduction of the energy consumption of the knee and the hip of the stance leg. We can see figure (9), for the robot with four-bar knees, the template torques/joint velocities is clearly oversized. The physical characteristics of the motors, weigth, inertia, length should be less for a four-bar knee.

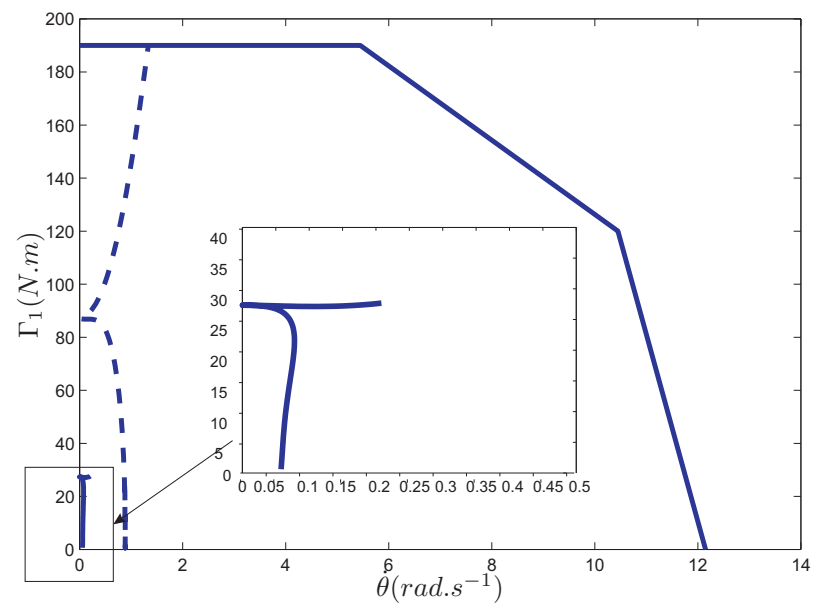

Fig. 9. Representation of the template of the maximum torque used for the knee joint. Evolution of the knee support leg torque in function of the articular velocity for the robot with four-bar knees (straight line) and for the robot with single axis knees (dashed line) for a step at $1 \mathrm{~m} / \mathrm{s}$.

Figure (10), we introduce a criterion on the evolution of the vertical position of the center of mass of the bipedal robot with:

$$
\Delta_{H}=\max \left(z_{g}\right)-\min \left(z_{g}\right)
$$

The evolution of criterion $\Delta_{H}$ is in function of the walking rate for both biped. We can see the four-bar knee reduces the vertical excursion of the center of mass that induces a reduction of the potential energy during the gait.

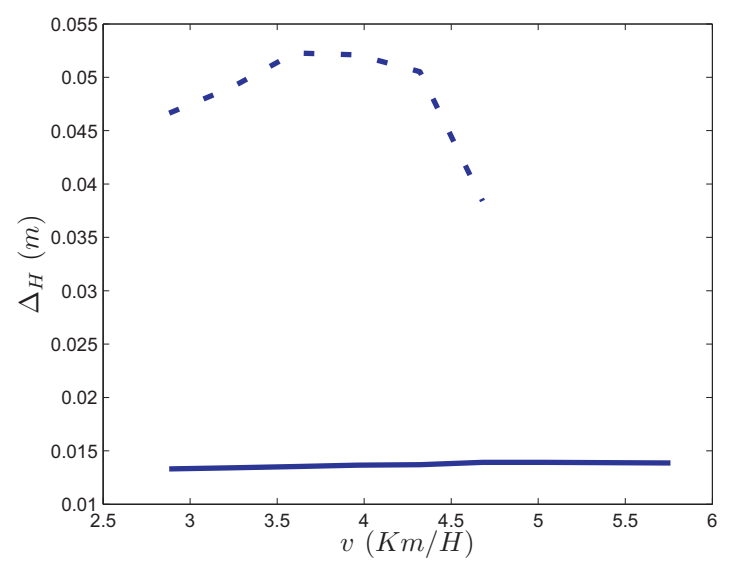

Fig. 10. Evolution of the vertical excursion of the center of mass in function of the walking rate for the biped with four-bar knees (straight line) and for the biped with single axis knees (dashed line).

From the profiles of the norm of the impulsive ground reaction, figure (11), we can conclude the four-bar knee reduces the impact forces. This result may be explained by the flexibility given by the four-bar structure. 


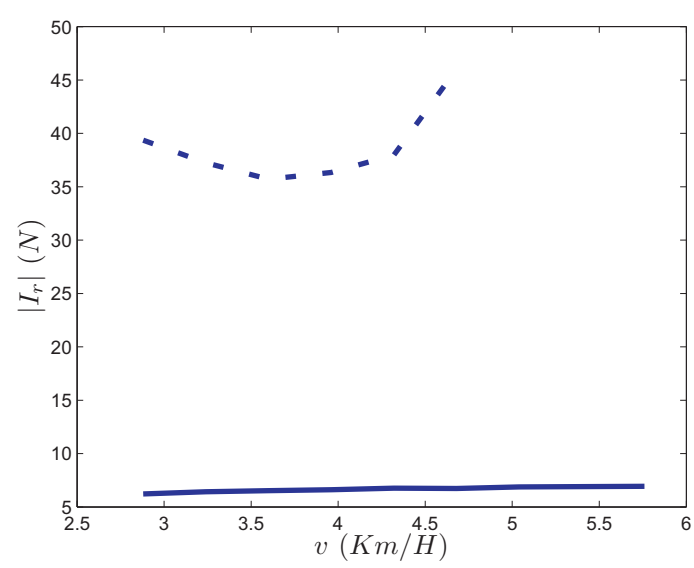

Fig. 11. Evolution of the norm of the impulsive ground reaction force in function of the walking rate for the biped with four-bar knees (straight line) and for the biped with single axis knees (dashed line).

\section{CONCLUSION}

In this paper we have studied two types of knee joints for a planar bipedal robot without feet. Due to the fact there is no feet, these bipeds are underactuated in single support phase. A single axis articulation, which uses on the most bipedal robots and a four-bar knee, which composes of a parallel structure. In both case, we have defined a parametric optimization problem to obtain a walking cyclic step for an under actuated bipedal robot. The steps are composed of single support phases and impulsive impacts. By solving of this optimization problem we have study the energy consumption of the biped for the two different knee joints. With the help of the definition of reference trajectories and this underactuated property we can see the influence of both structures on the orbital stability for the two bipeds.

The main advantage of the four-bar knee joint is to reduce the energy consumption but decreases the stability than a single axis joint. Furthermore, the four-bar knee reduces the vertical excursion of the body center of mass, and then minimizes the potential energy, and decreases the impulsive forces at the impact. In perspective of this work, an extension of this study in 3D could be done and a study of the effect of the four-bar knee on the foot clearance in the case of bipeds with feet.

\section{REFERENCES}

[1] C. Chevallereau and Y. Aoustin, "Optimal reference trajectories for walking and running of a biped robot," Robotica, vol. 19, no. 5, pp. 557-569, 2001.

[2] S. Miossec, "Contribution à la marche d'un bipède," Thèse de doctorat, Université de Nantes, Nantes, France, Novembre 2004.

[3] D. Tlalolini Romero, C. Chevallereau, and Y. Aoustin, "Comparison of different gaits with rotation of the feet for a planar biped," Robotics and Autonomous Systems, vol. 57, no. 4, pp. 371-383, Avril 2009.

[4] G. Bessonnet, S. Chesse, and P. Sardain, "Optimal Gait Synthesis of a Seven-Link Planar Biped," The International Journal of Robotics Research, vol. 23, no. 10-11, pp. 1059-1073, 2004.

[5] G. Bessonnet, P. Seguin, and P. Sardain, "A Parametric Optimization Approach to Walking Pattern Synthesis," The International Journal of Robotics Research, vol. 24, no. 7, pp. 523-536, 2005.

[6] P. Klein and P. Sommerfeld, Biomécanique des membres inérieurs : bases et concepts, bassin, membres inférieurs. Elsevier, 2008.
[7] D. Winter, Biomechanics and motor control of human movement. Wiley, 2005.

[8] J. Holden and S. Stanhope, "The effect of variation in knee center location estimates on net knee joint moments," Gait Posture, vol. 7, pp. 1-6, 1998.

[9] D. Subit, "Modélisation de la liaison os-ligament dans l'articulation du genou," Ph.D. dissertation, Université de la méditerranée - Aix Marseille II, 2004.

[10] L. Seddiki, "Développement et commande t-s d'une machine de rééducation des membres inférieurs en chaîne musculaire fermée," Ph.D. dissertation, Université de Reims Champagne Ardenne, 2008.

[11] K. Kaneko, F. Kanehiro, S. Kajita, H. Hirukawa, T. Kawasaki, M. Hirita, K. Akachi, and T. Isozumi, "Humanoid robot hrp-2," in Proceedings of the International Conference on Robotics and Automation 2004, 2004, pp. 1083-1090.

[12] C. Chevallereau, G. Abba, Y. Aoustin, F. Plestan, E. Westervelt, C. Canuddas-de Wit, and J. Grizzle, "Rabbit: a testbed for advanced control theory," IEEE Control Systems Magazine, vol. 23, no. 5, pp. 57-79, 2003.

[13] Y. Ogura, H. Aikawa, K. Shimomura, H. Kondo, A. Morishima, H. Lim, and A. Takanishi, "Development of a new humanoid robot wabian-2," in Proceedings of the IEEE International Conference on Robotics and Automation 2006, 2006, pp. 76-81.

[14] G. Gini, U. Scarfogliero, and M. Folgheraiter, "Human-oriented biped robot design : insights into the development of a truly antropomophic leg," in IEEE International Conference on Robotics and Automation, 2007, pp. 2910-2915.

[15] F. Wang, C. Wu, Y. Zhang, and X. Xu, "Design and implementation of coordinated control strategy for biped robot with heterogeneous legs," in IEEE International Conference on Mechatronics and Automation, 2007, pp. 1559-1564.

[16] T. Lu and J. O'Connor, "Lines of action and moment arms of the major force-bearing structures crossing the human knee joint: comparison between theory and experiment," Journal of Anatomy, vol. 189, no. 3, pp. 575-585, 1996.

[17] S. Dye, "An evolutionary perspective of the knee," The Journal of Bone and Joint Surgery, vol. 69, no. 7, pp. 976-983, 1987.

[18] W. Dempster, "Space requirements of seated operator," University of Michigan, Tech. Rep., 1955.

[19] M. Spong and M. Vidyasagar, Robot dynamics and control. John Wiley, New-York, USA, 1991.

[20] A. Formal'skii, Locomotion of Anthropomorphic Mechanisms. [In Russian], Nauka, Moscow, Russia, 1982.

[21] A. Grishin, A. Formal'sky, A. Lensky, and S. Zhitomirsky, "Dynamic Walking of a Vehicle With Two Telescopic Legs Controlled by Two Drives," The International Journal of Robotics Research, vol. 13, no. 2, pp. 137-147, 1994.

[22] Y. Aoustin and A. Formal'sky, "Control design for a biped reference trajectory based on driven angles as functions of the undriven angle," Journal of Computer and System Sciences International, vol. 42, no. 4, pp. 159-176, 2003.

[23] S. Miossec and Y. Aoustin, Dynamical synthesis of a walking cyclic gait for a biped with point feet, ser. Lecture Notes in Control and Information Sciences. Springer Berlin / Heidelberg, 2006, vol. 340, pp. 233-252.

[24] C. Chevallereau, A. Formal'skii, and D. Djoudi, "Tracking of a joint path for the walking of an under actuated biped," Robotica, vol. 22, no. 1, pp. 15-28, 2003.

[25] L. Fridman, Y. Aoustin, and F. Plestan, "A framework for stability analysis of singularly perturbated periodical nonlinear systems with impulse effects: application to a bipedal robot," in IEEE Conference on Decision and Control CDC, Cancun, MEXIQUE, 2008, pp. 52385243.

[26] P. Gill, W. Murray, and M. Wright, Practical optimization. London: Academic Press, 1981.

[27] M. Powell, Variable metric methods for constrained optimization, ser. Lecture Notes in Mathematics. Springer Berlin / Heidelberg, 1977, pp. 62-72.

[28] J. Bobrow, F. Park, and A. Sideris, Recent Advances on the Algorithmic Optimization of Robot Motion, ser. Lecture Notes in Control and Information Sciences. Springer Berlin / Heidelberg, 2006, vol. 340, pp. $21-41$.

[29] K. Mombaur, "Stability optimization of open-loop controlled walking robots," Ph.D. dissertation, University of Heidelberg, 2001. 